Research Paper:

\title{
Three-dimensional Anaglyph Indocyanine Green Video Angiography: A New Technology for Cerebrovascular Surgeries
}

\author{
Mohsen Nouri ${ }^{1,2^{*}}$ (D) Amir Azarhomayoun ${ }^{2}$ (D)
}

1. Department of Neurosurgery, Jamaica Hospital Medical Center, New York, USA.

2. Stone Lion Clinic, Gundishapour Academy of Neuroscience, Ahvaz, Iran.



Crtation: Nouri M, Azarhomayoun A. Three-dimensional Anaglyph Indocyanine Green Video Angiography: A New Technology for Cerebrovascular Surgeries. Iran J Neurosurg. 2021; 7(1):31-36. http://dx.doi.org/10.32598/irjns.7.1.3

doij: http://dx.doi.org/10.32598/irjns.7.1.3

\section{(c) (1) (\$)}

Article info:

Received: 18 Jun 2020

Accepted: 23 Oct 2020

Available Online: $01 \mathrm{Jan} 2021$

Keywords:

Technology, Angiography, Indocyanine green, Threedimensional

\section{A B S T RACT}

Background and Aim: Indocyanine Green (ICG) angiography has become the standard of practice in many centers managing cerebrovascular diseases. Though 3D stereoscopic recording of the surgeries has received widespread attention, there is a need for a technology to display and record real-time 3D ICG angiographies. In this study, we designed and constructed an ICG angiography camera to display the real-time 3D ICG angiographies.

Methods and Materials/Patients: Our project had three phases. In phase I, a handmade ICG camera was designed and constructed in our laboratory. The second phase included creating a 3D camera to display real-time images in 3D anaglyph format. In the last phase, we developed a 3D ICG camera to demonstrate 3D ICG angiographies in real-time.

Results: We successfully completed all three phases of the project and could display real-time 3D ICG angiography of a mouse mesenteric arteries, recorded it, and took pictures.

Conclusion: We proposed a method and proved its feasibility for producing a 3D ICG angiography camera to be mounted on the next generation of neurosurgical microscopes. 


\section{Highlights}

- Real-time 3D hand-made ICG angiography is applicable for displaying vessels.

- Real-time 3D ICG angiography helps with a better understanding of the vascular anatomy.

- Our constructed device achieved acceptable 3D image quality and is highly promising for further development.

\section{Plain Language Summary}

Indocyanine Green (ICG) angiography has become the standard of practice in many centers. we designed and constructed an ICG angiography camera to display real-time 3D ICG angiographies. Our project had three phases. We developed a 3D ICG camera to demonstrate 3D ICG angiographies in real-time. We successfully completed all three phases of the project. We proposed a method and proved its feasibility for producing a 3D ICG angiography camera to be mounted on the next generation of neurosurgical microscopes.

\section{Introduction}

eal-time three-dimensional stereoscopy/ $\mathbf{R}$ anaglyph display of the surgeries is a common practice enabling surgeons to have a better understanding of the surgical approach and improving the training of the residents and observers in the operation theatres [1]. Also, 3D anaglyph presentations are being popularized not only for teaching complicated neurosurgical anatomy (e.g., skull base) to medical students and residents [2, 3], but also for training young neurosurgeons in cerebrovascular surgeries [4].

Although intra-operative Indocyanine Green (ICG) Video Angiography (VA) has become an integral part of cerebrovascular surgeries $[5,6]$, there is no microscope-mounted technology to display real-time ICG-VA or to capture them for later demonstrations in 3D anaglyph format. In this article, we present our recently invented camera which displays and records 3D anaglyph ICG-VA, describe its technical details, and discuss its applications.

\section{Methods and Materials/Patients}

All animal experiments in this project were in accordance with Helsinki Declaration and followed the animal care guidelines. For each experiment, 5 Balb/C female mice (20-25 gr) were purchased from the Faculty of Veterinary Medicine, University of Tehran, Iran. Animals were kept inhouse with ad lib access to water and food. Anesthesia was induced with intraperitoneal injection of Ketamine $50 \mathrm{mg} /$ kg (Alfasan, Woerden, Holland) and Acepromazine $5 \mathrm{mg} /$ kg (Castran, Interchemie, Holland) and maintained with half-dose anesthetics at intervals if required. After midline laparotomy, mesenteric vessels of the small intestine were exposed and kept wet with normal saline. The ICG (SERB, Paris, France) was reconstituted with sterile water at the beginning of every experience. All ICG injections were given intravenously through the tail with an insulin syringe (1 $\mathrm{mg} / \mathrm{kg}$ ). After the procedure, the animal would be euthanized with an intraperitoneal dose of sodium pentobarbital 120 mg/kg (Sigma, MO, USA) while still under anesthesia.

The project had three phases. The first phase included four animal experiments with each requiring 2-3 mice. Experiments were performed at variable intervals after modifying the camera or troubleshooting an existing problem. In the second phase, we did not perform an animal experiment, and finally, in the third phase, we used only one mouse in a single experiment. In the first phase, an ICG-VA camera was designed and constructed in our laboratory which was described in detail elsewhere [7]. Briefly, a laser and beam projector (Roithner Lasertechnik $\mathrm{GmbH}, \mathrm{Vi}$ enna, Austria) was used to illuminate the surgical field with $780 \mathrm{~nm}$ wavelength rays to excite fluorescence in blood protein-bound ICG. The reflected fluorescence light by the ICG would be detected by a Charged Couple Device (CCD) surveillance camera (Sony Super HAD II CCD image sensor with 650TVL resolution, assembled in Iran) equipped with a long-pass optic filter (Marumi, Nagano, Japan) to detect lights at $830 \mathrm{~nm}$ wavelength and longer. In the second phase, a 3D camera was made consisting of two CCD surveillance cameras similar to the one in phase $I$. The centers of the two cameras were almost $7 \mathrm{~cm}$ apart and their axis had a 12-degree convergence similar to the human eyes while focusing on an object at $30 \mathrm{~cm}$. These two cameras were connected through two Easycap USB-2.0 video capture adapters (Easyday, China) to a laptop where two stereoscopy software namely Stereoscopic Multiplexer (Ver- 
sion 0.7, Austria) and Stereoscopic Player (Version 2.4.3, Austria) were used to digitally filter the input images and merge them to create real-time anaglyph red-cyan 3D images. In the last phase of the study, both CCD components of this 3D camera were equipped with $850 \mathrm{~nm}$ long pass optic filters (GREEN.L, China). Near infra-red laser (780 nm wavelength) and projector (775 nm wavelength light with short-pass optic filter $780 \mathrm{~nm}$ ) similar to the ones used in phase I, were used to illuminate the surgical field.

\section{Results}

As published previously, the camera in the first phase of the study recorded real-time 2D ICG-VA (Figure 1A). Simultaneously, another CCD camera without any filter would record the regular surgical field [7]. In the second phase of the study, the 3D camera was used to take pictures and videos of a plastic skull model which showed acceptable 3D qualities while being watched by red-cyan anaglyph glasses (figures not shown).

In the last phase, after inducing anesthesia and exposing mesenteric vessels, the projector and laser were used to illuminate the surgical field. In addition to the 2D camera, the $3 \mathrm{D}$ camera recorded the surgical field throughout the procedure (Figure 1B). Intravenous ICG was injected and the $3 \mathrm{D}$ anaglyph real-time images could be observed with red-cyan glasses (Figure $1 \mathrm{C}$ ).

\section{Discussion}

Real-time display or video recording of surgeries in 3D anaglyph mode has gained popularity and attention in many centers $[3,4]$. Though we lack solid data, it has been suggested that 3D displays and videos may help young neurosurgeons and trainees with surface detection and depth judgment and therefore, better decision making during brain surgeries [8]. Newer generations of neurosurgical microscopes are equipped with 3D cameras $[9,10]$. Also, some centers use microscope- or rigmounted 3D cameras for research or educational purposes in their cadaver laboratories for brain or skull base dissections $[11,12]$ as they believe it helps with a better understanding of complex neurosurgical anatomy and approaches. Our proposed technology may help us with three dimensional understanding of vascular pathologies intra-operatively and education of young neurosurgeons in workshops and presentations.

Performing 3D ICG angiographies had some obstacles. Available cameras use red and blue/cyan optic filters to create two images (i.e., left and right) of the anaglyph format. As the reflected light from protein-bound ICG is
$900 \mathrm{~nm}$, this light is filtered by red or cyan filters and can not be detected by the camera. Our solution to solve this problem was to use post-imaging electronic filtering rather than usual optic filtering. For this purpose, the regular ICGVAs from two cameras, which are in white color, would be filtered to either red or cyan and merged at the same time as left and right stereoscopic images by the software. Theoretically, this can also be achieved by commercially available hardware to synchronize the images such as multiplexer Hi5-3D (AJA video systems, USA) but we have not used it in our experiments because of unavailability in our market. Another alternative is using a 3D stereo lens Kula Deeper (Kula 3D Ltd, Iceland), and its software Kulacode V0.98 (Kula 3D Ltd, Iceland). In this method, only one camera and a near-infrared filter are enough to take pictures. On the other hand, it has some limitations. The width of the final 3D image would be half of the 2D images taken by the camera. Also, it requires post-imaging software processing which makes real-time display impossible. Finally, in our experience, it is more appropriate for distant and big objects rather than a small surgical field close to the surgeon. For small and close objects (e.g. a mouse intestine), we would require magnifying lenses to achieve better quality images. Considering the limitations, we found this method more appropriate for photography (e.g. from cadavers) rather than live display and by using a high-quality Digital Single-Lens Reflex (DSLR) camera instead of CCD surveillance cameras.

Considering the high costs of 3D recording systems such as MCC-3000MT (Sony Electronics Inc., NJ, USA), there are many reports even from the developed countries who have created handmade or custom-built 3D cameras according to their needs [13]. There is a similar scenario with cost-prohibitive ICG-cameras where handmade cameras are being used for clinical or research purposes [14]. When ICG-angiography was finding its way into surgical and neurosurgical theatres, there were many reports of handmade systems [15] and later, commercially available manual cameras became popular [16] but, finally, these cameras became an integral part of modern microscopes $[9,10]$. We believe that by using our suggested method, leading industries on the ground can integrate 3D ICG cameras into neurosurgical microscopes in the very near future. Our constructed ICG camera is currently being used in the laboratory for the development of animal models of cerebrovascular diseases and a flow measuring software [7]. This presented 3D ICG camera was also a part of our ongoing projects.

Major limitations of our camera were due to inaccessibility to the required components including cameras, lenses, filters, lasers, light resources, and software in and from 

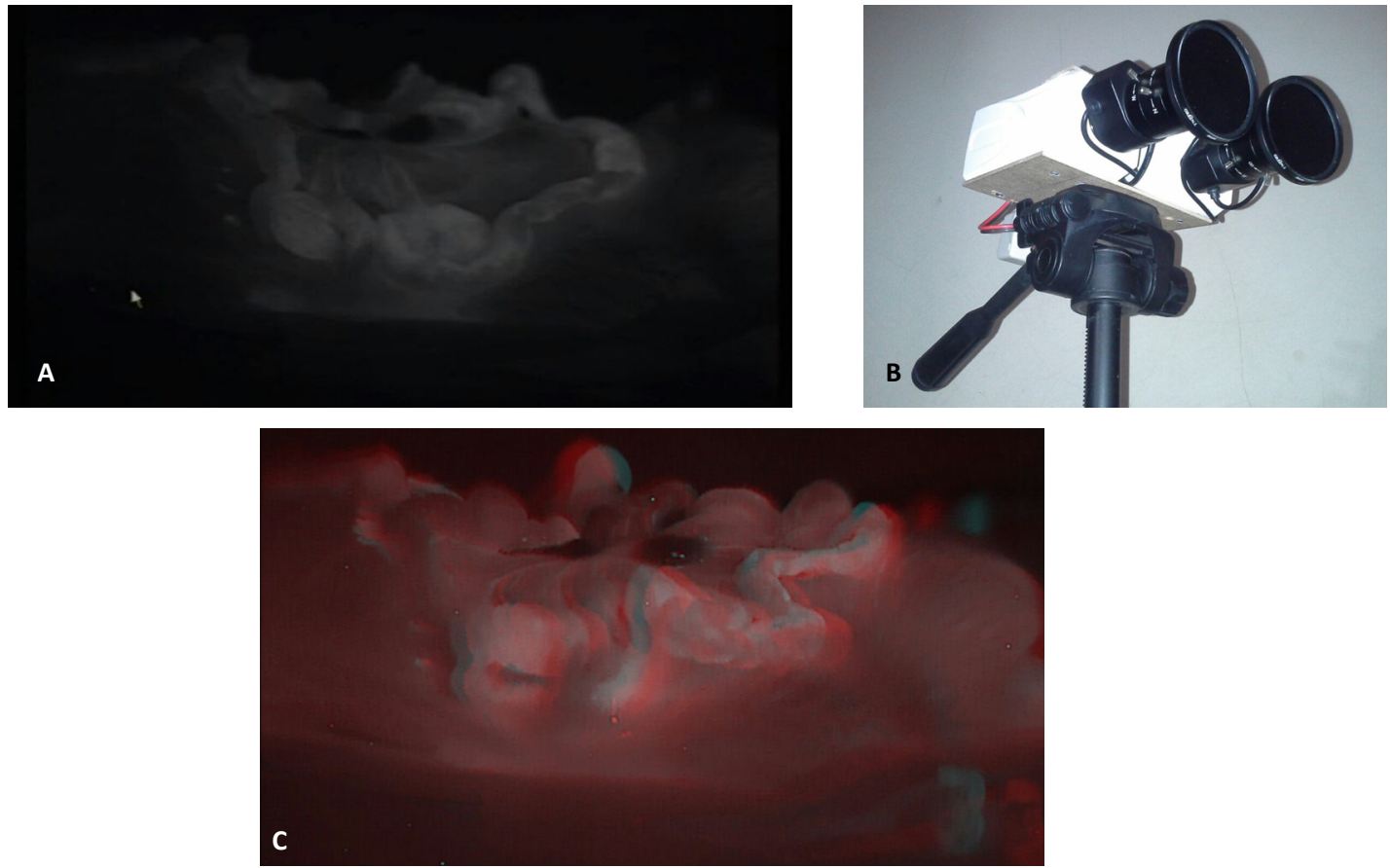

Figure 1. Two- and three-dimensional Indocyanine Green (ICG) video angiographies and the 3D camera

A: Two-dimensional ICG angiography of the mouse mesenteric arteries in late arterial/perfusion phase; B: Real-time 3D ICG angiography of the same arteries but from a slightly different angle; C: Three-dimensional ICG angiography system which consists of two surveillance cameras and lenses equipped with long pass $850 \mathrm{~nm}$ optic filters.

our country. By replacing the cameras with medical-grade near-infrared cameras, the resolution of the pictures will be enhanced enormously. Also, illuminating the whole surgical field with high-power lasers and using high-precision optical filters will improve image contrast and visibility of the ICG reflecting structures (i.e. vessels) in more detail. On the other hand, we could not use synchronizers which are necessary for 3D real-time display or video recording to achieve the optimum quality. The sense of 3D imaging will be improved dramatically after implementing all these changes to enhance the image quality. In spite of all the limitations, our constructed device achieved acceptable 3D image quality and is highly promising for further development.

\section{Conclusion}

We presented our report of 3D anaglyph ICG angiography. This technology may help cerebrovascular neurosurgeons with a better understanding of the vascular anatomy and depth perception during surgery and assist neurosurgery residents and fellows to sense operations in three dimensions and improve their concept and imagination of the neurovascular anatomy.

\section{Ethical Considerations}

\section{Compliance with ethical guidelines}

There were no ethical considerations to be considered in this research.

\section{Funding}

The project was financed by the Gundishapour Academy of Neuroscience and no external fund was received for any part of the study.

\section{Authors' contributions}

Conception and design: Mohsen Nouri; Data collection: Amir Azarhomayoun, Mohsen Nouri; Data analysis and interpretation: Amir Azarhomayoun, Mohsen Nouri; Drafting the article: Amir Azarhomayoun; Critically revising the article: Mohsen Nouri; Reviewing submitted version of manuscript: Amir Azarhomayoun, Mohsen Nouri.

\section{Conflict of interest}

The authors declared no conflict of interest. 


\section{References}

[1] Christopher LA, William A, Cohen-Gadol AA. Future directions in 3-dimensional imaging and neurosurgery: Stereoscopy and autostereoscopy. Neurosurgery. 2013; 72(Suppl 1:131-8. [DOI:10.1227/NEU.0b013e318270d9c0] [PMID]

[2] Clark AD, Guilfoyle MR, Candy NG, Budohoski KP, Hofmann R, Barone DG, et al. Stereoscopic three-dimensional neuroanatomy lectures enhance neurosurgical training: Prospective comparison with traditional teaching. World Neurosurg. 2017; 108:917-23. [DOI:10.1016/j.wneu.2017.09.019] [PMID]

[3] Kockro RA, Amaxopoulou C, Killeen T, Wagner W, Reisch $\mathrm{R}$, Schwandt E, et al. Stereoscopic neuroanatomy lectures using a three-dimensional virtual reality environment. Annals of Anatomy. 2015; 201:91-8. [DOI:10.1016/j.aanat.2015.05.006] [PMID]

[4] Rubino PA, Bottan JS, Houssay A, López ES, Bustamante J, Chiarullo M, et al. Three-dimensional imaging as a teaching method in anterior circulation aneurysm surgery. World Neurosurg. 2014; 82(3-4):e467-74. [DOI:10.1016/j. wneu.2013.02.065] [PMID]

[5] Riva M, Amin-Hanjani S, Giussani C, De Witte O, Bruneau $M$. Indocyanine Green videoangiography in aneurysm surgery: Systematic review and meta-analysis. Neurosurgery. 2018; 83(2):166-80. [DOI:10.1093/neuros/nyx387] [PMID]

[6] Kato Y, Yamada Y, Sadato A, Nouri M, Cherian I, Tanaka T, et al. Intraoperative anatomical and hemodynamic analysis of intracerebral arteriovenous malformations by semi-quantitative color-coded indocyanine Green videoangiography. Asian Journal of Neurosurgery. 2017; 12(4):638-43. [DOI:10.4103/ ajns.AJNS_62_14] [PMID] [PMCID]

[7] Azarhomayoun A, Nouri M. In-house handmade camera for indocyanine Green video angiography. Iranian Journal of Neurosurgery. 2018; 4(1):13-8. [DOI:10.32598/irjns.4.1.13]

[8] Heath MD, Cohen-Gadol AA. Intraoperative stereoscopic 3D video imaging: Pushing the boundaries of surgical visualisation and applications for neurosurgical education. British Journal of Neurosurgery. 2012; 26(5):662-7. [DOI:10.3109/026 88697.2012.672057] [PMID]

[9] Leica Microsystems. Save time for 2D and 3D analysis. Leica Microsystems; 2021. Available from: https://www.leica-microsystems.com/products/surgical-microscopes/neurosurgery-spine/details/product/truevision-integrated-3d/

[10] Integrated Digital Optics. Medical Technology | for healthcare professionals; 2021. Available from: https://www.zeiss. $\mathrm{com} / \mathrm{meditec} / \mathrm{us} /$ products/neurosurgery/surgical-microscopes/kinevo-900.html

[11] Weill Cornell Brain and Spine Center. The skull base lab. Available from: http://weillcornellbrainandspine.org/skullbase-lab

[12] Skull Base Lab. Skull base and cerebrovascular laboratory [Internet]. 2021. Available from: http://skullbaselab.ucsf.edu/

[13] Lee B, Chen BR, Chen BB, Lu JY, Giannotta SL. Recording stereoscopic 3D neurosurgery with a head-mounted 3D camera system. British Journal of Neurosurgery. 2015; 29(3):371-3. [DOI:10.3109/02688697.2014.997664] [PMID]
[14] Liu Y, Bauer AQ, Akers WJ, Sudlow G, Liang K, Shen D, et al. Hands-free, wireless goggles for near-infrared fluorescence and real-time image guided surgery. Surgery. 2011; 149(5):68998. [DOI:10.1016/j.surg.2011.02.007] [PMID] [PMCID]

[15] Holm C, Tegeler J, Mayr M, Becker A, Pfeiffer UJ, Muhlbauer W. Monitoring free flaps using laser-induced fluorescence of indocyanine Green: A preliminary experience. Microsurgery. 2002; 22(7):278-87. [DOI:10.1002/micr.10052] [PMID]

[16] Woitzik J, Horn P, Vajkoczy P, Schmiedek P. Intraoperative control of extracranial-intracranial bypass patency by near-infrared indocyanine Green videoangiography. Journal of Neurosurgery. 2005; 102(4):692-8. [DOI:10.3171/ jns.2005.102.4.0692] [PMID] 
This Page Intentionally Left Blank 